\title{
High resolution MRI for preoperative work-up of neonates with an anorectal malformation: a direct comparison with distal pressure colostography/fistulography
}

\author{
Maarten G. Thomeer ${ }^{1} \cdot$ Annick Devos $^{2} \cdot$ Maarten Lequin $^{2} \cdot$ Nanko De Graaf $^{2}$ • \\ Conny J. H. M. Meeussen ${ }^{3} \cdot$ Morteza Meradji $^{2} \cdot$ Ivo De Blaauw $^{3} \cdot$ Cornelius E. J. Sloots $^{3}$
}

Received: 23 September 2014 / Revised: 30 March 2015 / Accepted: 8 April 2015 /Published online: 23 May 2015

(C) The Author(s) 2015. This article is published with open access at Springerlink.com

\begin{abstract}
Objective To compare MRI and colostography/fistulography in neonates with anorectal malformations (ARM), using surgery as reference standard.

Methods Thirty-three neonates ( 22 boys) with ARM were included. All patients underwent both preoperative highresolution MRI (without sedation or contrast instillation) and colostography/fistulography. The Krickenbeck classification was used to classify anorectal malformations, and the level of the rectal ending in relation to the levator muscle was evaluated.

Results Subjects included nine patients with a bulbar rectourethral fistula, six with a prostatic recto-urethral fistula, five with a vestibular fistula, five with a cloacal malformation, four without fistula, one with a H-type fistula, one with anal stenosis, one with a rectoperineal fistula and one with a bladderneck fistula. MRI and colostography/fistulography predicted anatomy in $88 \%(29 / 33)$ and $61 \%(20 / 33)$ of cases, respectively $(p=0.012)$. The distal end of the rectal pouch was correctly predicted in $88 \%(29 / 33)$ and $67 \%(22 / 33)$ of cases, respectively $(p=0.065)$. The length of the common channel in cloacal malformation was predicted with MRI in all $(100 \%, 5 / 5)$ and in $80 \%$ of cases (4/5) with colostography/fistulography.
\end{abstract}

Maarten G. Thomeer

m.thomeer@erasmusmc.nl

1 Department of Radiology, Erasmus MC, PO Box 2040, 's Gravendijkwal 230, 3000 CA Rotterdam, The Netherlands

2 Department of Pediatric Radiology, Erasmus MC, Rotterdam, The Netherlands

3 Department of Pediatric Surgery, Erasmus MC, Rotterdam, The Netherlands
Two bowel perforations occurred during colostography/ fistulography.

Conclusions MRI provides the most accurate evaluation of ARM and should be considered a serious alternative to colostography/fistulography during preoperative work-up.

Key Points

- High-resolution MRI is feasible without the use of sedation or anaesthesia.

- MRI is more accurate than colostography/fistulography in visualising the type of ARM.

- MRI is as reliable as colostography/fistulography in predicting the level of the rectal pouch.

- Colostography/fistulography can be complicated by bowel perforation.

Keywords MRI · Anorectal malformation · Fistulography · Colostography $\cdot$ Neonates
Abbreviations
MRI Magnetic resonance imaging
ARM Anorectal malformation

\section{Introduction}

Anorectal malformations (ARMs) are relatively rare congenital anomalies, with a reported incidence of around 3:10,000 live births [1]. The spectrum of anomalies ranges from mild forms, with a stenosis of the anus in its normal position, to more complex cases, with an aberrant location of the colonic outlet in the urogenital tract.

All occlusive and near-occlusive ARMs are treated soon after birth with a diverting colostomy in order to maintain gastrointestinal transit. Later, but preferably in the neonatal period, 
a reconstruction is planned with the main aim of achieving definitive bowel control and acceptable quality of life [2].

Accurate recognition of the different subtypes of ARM is essential for further operative management. Although several classification systems for ARMs have been proposed, the Krickenbeck classification-based on the presence/ absence of a fistula and its location - is the most widely accepted today $[2,3]$. The posterior sagittal anorectoplasty (PSARP), as described by Pena et al., is a commonly used surgical technique for anorectal malformations and requires a perineal approach. The technique can be used when the fistula ends below or at the level of the pelvic floor (levator muscle) [4]. Higher positioned fistulas (mainly the rectovesicular or prostatic recto-urethral) need a laparoscopic or laparotomic approach (LAARP) [2]. In cases of longer fistulas ending below the pelvic floor but with a distal rectum ending higher above the pelvic floor, the PSARP may not be sufficient to complete the reconstruction, and a laparoscopy or laparotomy may also be required. Preoperative imaging is thus mandatory for the surgeon deciding on the best surgical approach.

The most commonly used visualization technique for ARMs is high-pressure augmented colostography/fistulography. An additional micturating cystogram is often carried out simultaneously to further exclude associated urogenital anomalies [5].

An important advantage of using colostography is the possibility to apply controlled pressure in the distal colon in order to differentiate a normal colon from a distal fistula. On the other hand, the main disadvantages of this procedure include a risk of perforation, especially in boys, and the effects of radiation in the newborn. In relation to ARMs and the high rate of associated anomalies in the pelvis, another disadvantage of this technique is that it does not show any other anatomical structures of the pelvic floor, such as the levator muscle or spinal canal. This will influence the planning of surgery and the final prognosis.

Although magnetic resonance imaging (MRI) has been proposed as an alternative, effectively circumventing the radiation issue, literature is still sparse and dated [6-12]. MRI in the first month after birth is often difficult due to the need for very high resolution imaging in order to discern very small pelvic structures. Proposed use of a head coil rather than a phased array coil does not solve the problem of a resolution that is often greater than $3 \mathrm{~mm}$, which in our opinion is still suboptimal in neonates [10]. Another group described the use of MRI with local instillation of contrast in different orifices [6, 13]. While high-quality images can be produced using this technique, it is still relatively invasive and requires sedation.

We now propose a variant of early MRI using high resolution images (voxel size of $0.06 * 0.04 * 0.1-0.2 \mathrm{~cm}$ ), without instillation of contrast and without sedation or anaesthesia.
The question we will address is whether this new MR technique improves visualization of the anatomy in anorectal malformation compared to contrast colon studies, thus allowing optimised surgical planning and improved outcomes. The study design is a prospective comparison of MRI with classical pressure augmented colostography/fistulography in anorectal malformation patients, using final operative results as the reference gold standard.

\section{Materials and methods}

Between 2008 and the first half of 2014, all patients born in the Erasmus University Medical Center (Erasmus MC) in Rotterdam, the Netherlands with anorectal malformations and who were in their neonatal period (up to 4 months after birth) were prospectively included when visualization studies were indicated. In case of colonic outflow obstruction, the patients underwent a split diverting colostomy soon after birth and before imaging (28 patients). Patients were consecutively scanned with colostography/fistulography, cystography and MRI before reconstructive surgery. The study was approved by the Erasmus MC institutional review board and informed consent was obtained from the parents or the guardian.

All neonates were operated based on the combination of colostography/fistulography and MRI findings, and the surgical approach was either a PSARP or LAARP, depending on the classification proposed by the radiologists and the clinical findings.

\section{Imaging techniques}

\section{MRI}

All included patients underwent MRI using a $1.5 \mathrm{~T}$ unit (General Electrics, Signa, Milwaukee, WI). Parents were asked to stay in the MRI room during the examination. No analgesic was used and patients were bottle-fed immediately prior to the procedure. A one-channel, standard knee coil (5" round GP coil) was placed posterior to the anus. The patient and coil were then enveloped in a vacuum cushion in order to suppress patient movements.

Scans were started with a T2-weighted (T2W) single-shot fast spin echo [SSFSE, slice thickness $=3 \mathrm{~mm}$; repetition time/echo time $(\mathrm{TR} / \mathrm{TE})(\mathrm{msec})=$ minimum/90; flip angle $=$ $90^{\circ}$ ] in three directions, with a matrix of $256 \times 224$.

This was followed by minimally a $\mathrm{T} 2 \mathrm{~W}$ fast recovery fast spin echo (FRFSE, slice thickness $=1-2 \mathrm{~mm}, 3500 / 80$, $90^{\circ}$ ) with a matrix of $512 \times 256$, a field of view (FOV) of $20 \mathrm{~cm}$ and phase FOV of 0.8 . The number of acquisitions was 4 and the echo train length was 21 , with a low 
bandwidth of 31.25. This was performed with and without fat suppression in the axial and sagittal planes. The voxel size was $0.06 \times 0.04 \times 0.1 / 0.2 \mathrm{~cm}$. The duration of the MRI investigation was around $30 \mathrm{~min}$. When necessary, some of the sequences were repeated (mainly due to movements of the child).

\section{Colostography or fistulography}

Pressure-augmented colostography was performed by insertion of a Foley catheter in the distal rectal pouch and by enema of the distal colorectal pouch with water-soluble non-ionic contrast media [14]. The addition of air insufflation with controlled pressure was used to obtain an optimal distension of the rectal pouch resulting in imaging of possible fistular communications. When the neonate did not have a colostomy, the distal fistula was injected with contrast agent. A micturating cystogram was performed in all patients in order to exclude associated urological abnormalities.

\section{Image evaluation}

The MRI studies were prospectively and independently analysed by two readers (two experienced staff members of paediatric radiology and abdominal radiology, A and B), blinded to the results of surgery. Information regarding the anal opening, which was clinically visible, was provided to both readers in order to imitate clinical reality [15]. Information from other diagnostic tools was not available during the reading sessions.

Colostography/fistulography was prospectively and independently analysed by two other readers (two experienced staff members of paediatric radiology, $\mathrm{X}$ and $\mathrm{Y}$ ), also blinded to MRI or surgical results. The same information was given as for the MRI readings.

Firstly, readers were asked to classify the imaging findings according to the Krickenbeck classification [3, 16]. Secondly, they were asked to evaluate the location of the transition of the normal rectal mucosa into the fistula. The location of the transition was classified as above the pelvic floor, at the level of the pelvic floor or below the pelvic floor [2]. Since no pressure was applied during MRI to dilate the proximal colon, differentiation between normal colon and fistula was based on the layered aspect of the bowel segment. If the different layers (mucosa, submucosa and muscularis) were discernable, it was classified as normal bowel that could be used for anastomosis. If there were no layers visible, it was classified as a fistula. Using colostography/ fistulography, the non-dilated part of the distal bowel was classified as a fistula.
In patients with a cloacal malformation, the length of the common channel was measured as the length between the rectal fistula, at the point where urethra and vagina connect, and the rectum to the distal channel orifice at the perineum.

Finally, the visibility of sacrococcygeal bones was described based on the sagittal T2- weighted MRI data. This was only performed using MRI, since it was difficult to differentiate the sacral from the lumbar bones with colostography/fistulography (due to the overpenetration of $\mathrm{X}$-rays). Additional urogenital anomalies visible on MRI were described, and complications during the investigations (MRI and colostography/fistulography) were also reported.

\section{Reference standard}

Reconstructive surgery was performed using the PSARP (perineal procedure) according to Pena et al. [4] or the LAARP technique (laparoscopic procedure) according to Georgeson et al. [17]. A technique was chosen based on review of the visualization studies at the level of the rectal pouch and the anatomy of the fistula.

During surgery, the anatomy of the fistula was noted according to the Krickenbeck classification. Precise measurement of the length of the rectal fistula was not attempted because the fistula was often conical in presentation, making it difficult to state where the fistula began and the rectum ended. Instead, the level of rectal pouch (above, on or below the pelvic floor) was noted. The combination of findings of surgery, urethroscopy and colostography/fistulography imaging was used as a reference standard for the measurement of the length of the common channel in case of a cloacal malformation, since direct measurement was not always feasible.

\section{Statistical analysis}

McNemar's test was used to compare the proportion of correct diagnoses (against the reference standard described above) made with either MRI or conventional fluoroscopic studies. $p$ values of less than 0.05 were considered significant. Interobserver agreement was assessed using the kappa value $(\mathrm{k})$. A $\mathrm{k} \leq 0.20$ was interpreted as slight agreement; $0.21-0.40$, fair agreement; 0.41-0.60, moderate agreement; $0.61-0.80$, substantial agreement; and $\geq 0.81$, almost perfect agreement [18]. All statistical analyses were performed using SPSS 16.0 (SPSS, Chicago, ILL).

\section{Results}

Thirty-six patients (22 boys and 14 girls) were admitted for anorectal malformation in the postnatal period. Of these, three were excluded, since only one examination was performed 
(either MRI or colostography/fistulography). Ages varied from the neonatal period up to four months. Twenty-eight patients underwent pressure, augmented colostography and five underwent distal fistulography based on presence of a sigmoidostomy. One MRI and four colostographies/ fistulographies were of insufficient quality. Findings included nine patients with a bulbar recto-urethral fistula, six with a prostatic recto-urethral fistula (Fig. 1), five with a vestibular fistula (Fig. 2), five with a cloacal malformation, four with no fistula, one with a H-type fistula, one with anal stenosis, one with a rectoperineal fistula, and one with a complex bladder fistula. Compared to surgery, MRI or colostography/ fistulography correctly predicted anatomy in $88 \%(29 / 33)$ and $61 \%(20 / 33)$ of cases, respectively $(p=0.012)$ (Table 1$)$. The distal end of the rectal pouch was correctly predicted in $88 \%(29 / 33)$ and $67 \%(22 / 33)$ of cases, respectively ( $p=$ $0.065)$. The length of the common channel in cloacas was correctly predicted by MRI in all cases $(5 / 5)$ and by colostography/fistulography in four out of five cases.

The inter-reader agreement of the two readers for Krickenbeck classifications with MRI was perfect (kappa of 1.0), whereas the kappa was 0.75 (substantial agreement) for colostography/fistulography. The inter-reader agreement of the two readers determining the position of the rectal pouch with MRI was perfect (kappa of 1.0), while colostography/ fistulography showed a corresponding kappa of only 0.58 (moderate agreement).

MRI failed to make the correct diagnosis in four cases: a rectovaginal $\mathrm{H}$-fistula, an anal stenosis, a vestibular fistula (insufficient image quality) and a bulbar recto-urethral fistula (closed bulbar recto-urethral fistula misdiagnosed as no fistula with rectal pouch below the pelvic floor).

Colostography/fistulography was incorrect in 13 cases: five bulbar recto-urethral fistulas [four misinterpreted as prostatic urethral fistulas (Fig. 3)], three with no fistula, two prostatic urethral fistulas [one misinterpreted as an rectobulbar rectourethra fistula, one uninterpretable (Fig. 1)], one cloaca, one rectovaginal $\mathrm{H}$-fistula, and one anal stenosis.
Fig. 1 a-h A 2-month-old boy with proven recto-bladder neck fistula. a represents a midsagittal view through the pelvis (T2-weighted fast spin echo sequence; slice thickness $1.5 \mathrm{~mm}$ ) with s-form of the distal rectal segment which enters the prostate from posterior. The axial MRI slices (b-g) (T2-weigthed fat-suppressed fast spin echo sequence; slice thickness $1.5 \mathrm{~mm}$ ) are shown from higher to lower levels in the pelvis. The rectum turns in a fistula (pink) with a short transprostatic course. This fistula ends in the bladder neck (yellow), which turns in the urethra (black). Although on first sight of a complex case, all elements could easily be discerned by both readers based on a combination of axial and sagittal views. On the other hand, correct analysis of colostography (h) images was found to be impossible, mainly due to overlapping contrast opacities in all directions

The level of rectal pouch was misinterpreted in four cases using MRI compared to surgery: one vestibular fistula with a fistula at the level of the pelvic floor was misinterpreted as a lower level fistula, one bulbar level with an obliterated fistula at the pelvic floor was misinterpreted as no fistula with a rectal pouch below the pelvic floor, one bulbar fistula with a rectal pouch on the level of the pelvic floor was misinterpreted as a bulbar fistula with a rectal pouch above the pelvic floor, and finally, one vestibular fistula with a fistula below the pelvic floor could not be interpreted with MRI. The reasons for misinterpretation of the level of rectal pouch when colostography/ fistulography was used were diverse.

The failure of colostography/fistulography to determine the length of the common channel in a cloaca was due to an uninterpretable image.

MRI showed genitourinary anomalies in 12 patients, including uterus anomalies in three, pelvic cysts in four, split prostate in four, atrophic testis in one and utriculus in one patient. Uterine anomalies included a uterus didelphys and a uterus bicornis. The pelvic cysts consisted of two presacral cysts, one between the urethra and vagina, and one adjacent to the prostate.

Two bowel perforations occurred, both during colostography/fistulography. One event occurred under augmented pressure used to visualize a possible fistula. The
Table 1 Total number of correct diagnoses based on MRI or colostography/fistulography. The Krickenbeck classification was used to classify malformations. Perioperative findings were used as a reference standard. The total number of correct diagnoses for the two preoperative investigations differed significantly $(p=0.012)$

\begin{tabular}{llll}
\hline Krickenbeck classification & Number of patients & MRI & Colostography/fistulography \\
\hline Bulbar recto-urethral fistula & 9 & $8(89 \%)$ & $6(75 \%)$ \\
Prostatic recto-urethral fistula & 6 & $6(100 \%)$ & $3(50 \%)$ \\
Vestibular fistula & 5 & $4(80 \%)$ & $3(60 \%)$ \\
Cloacal malformation & 5 & $5(100 \%)$ & $4(80 \%)$ \\
No fistula & 4 & $4(100 \%)$ & $3(75 \%)$ \\
H-type fistula & 1 & $0(0 \%)$ & $0(0 \%)$ \\
Anal stenosis & 1 & $0(0 \%)$ & $0(0 \%)$ \\
Rectoperineal fistula & 1 & $1(100 \%)$ & $1(100 \%)$ \\
Bladder neck fistula & 1 & $1(100 \%)$ & $0(0 \%)$ \\
Total & 33 & $29(88 \%)$ & $20(61 \%)$ \\
\hline
\end{tabular}

( ): percentage of correct diagnoses 


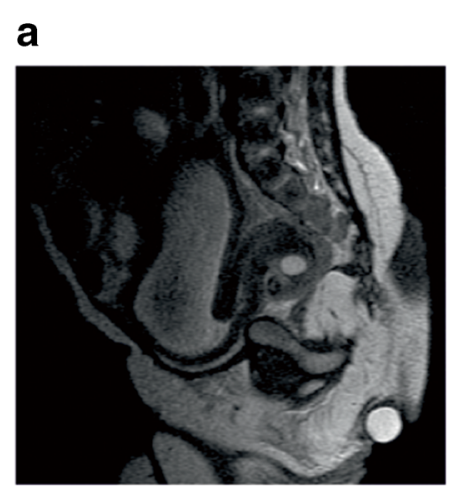

$\square$ Bones

Bowel

$\square$ Marker

Prostatic cyst

- Urethra
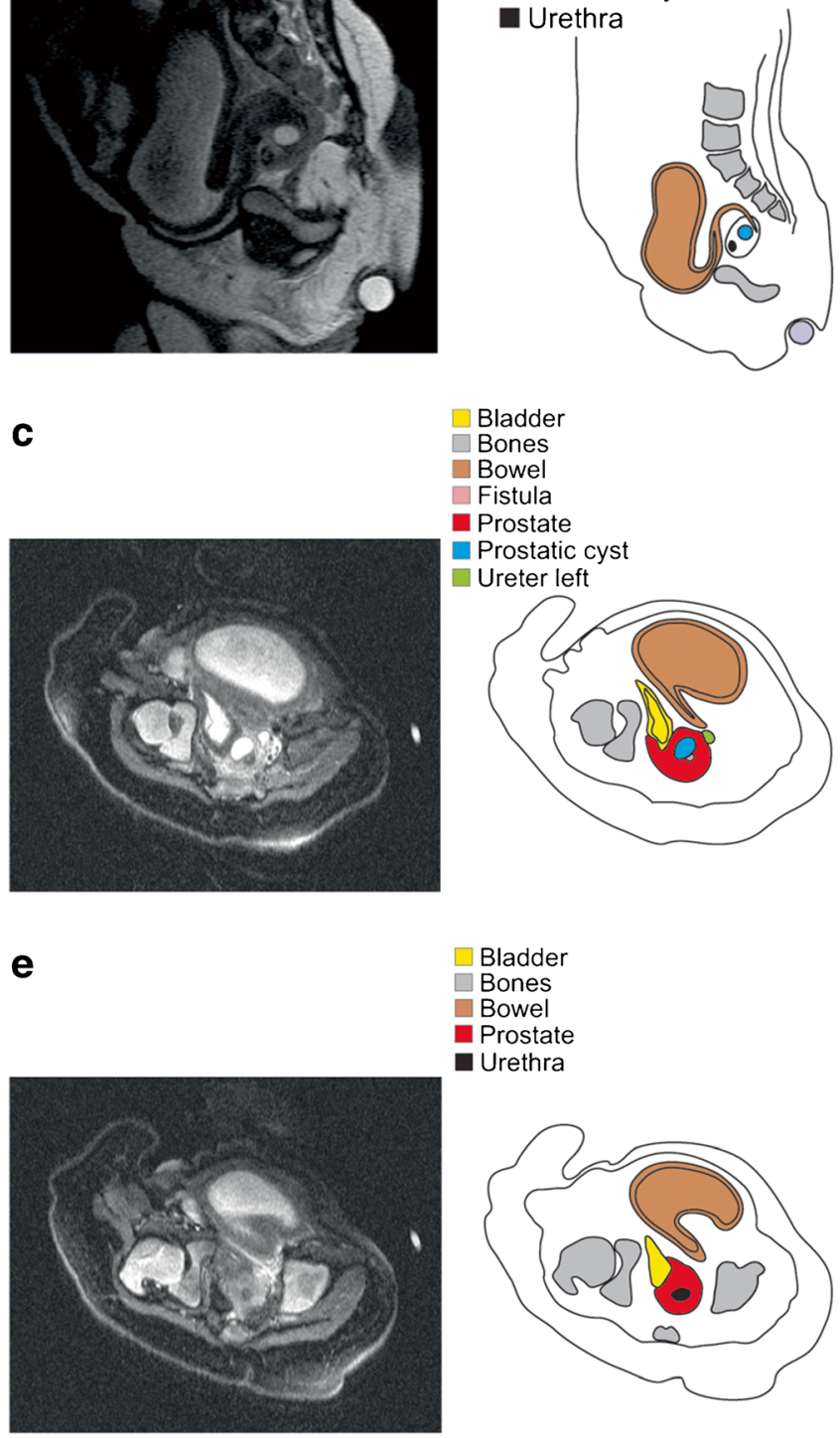

$\square$ Bladder

$\square$ Bones

- Prostate

- Urethra

g

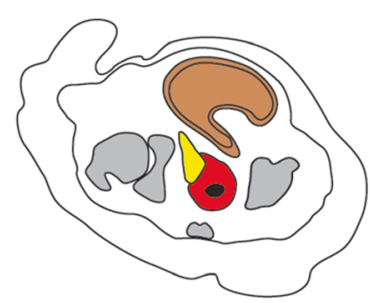

$\square$ Bones

Penis (bulbus cavernosum)

- Urethra
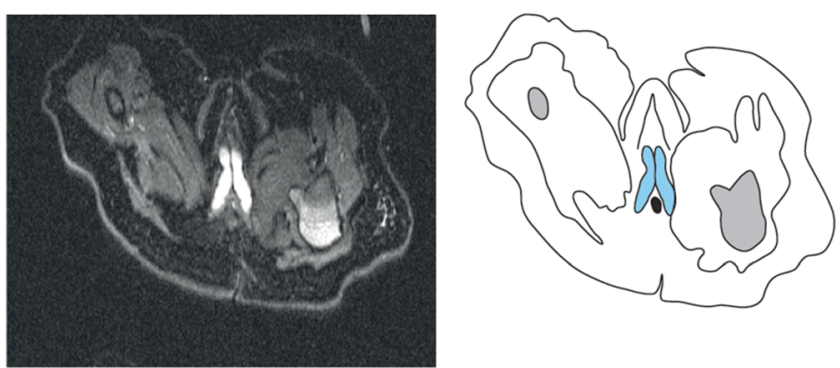

b

$\square$ Bladder
Bones
Bowel
Prostate
Ureter left
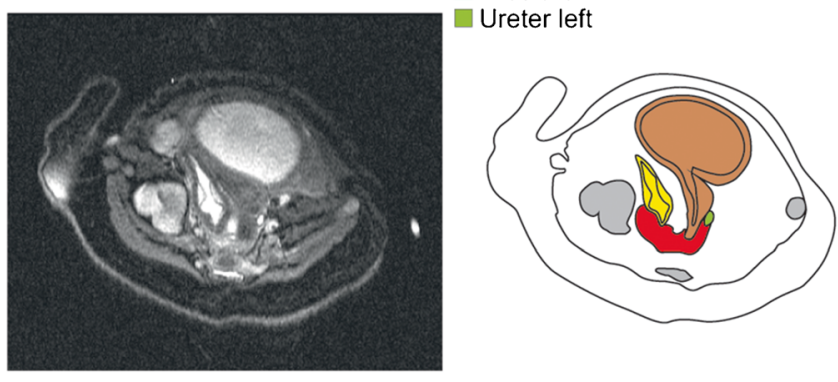

d

$$
\begin{aligned}
& \square \text { Bladder } \\
& \square \text { Bones } \\
& \text { Bowel } \\
& \square \text { Fistula } \\
& \square \text { Prostate }
\end{aligned}
$$

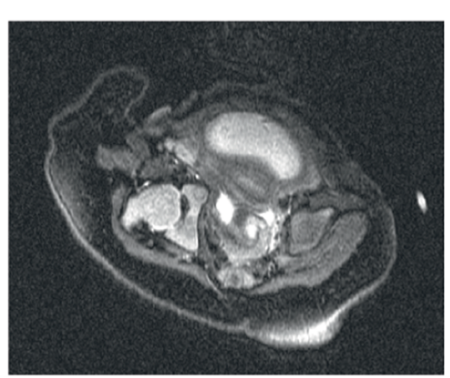

Prostatic cyst

Ureter left

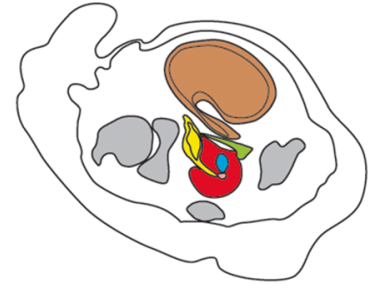

f

$\square$ Bones

Penis (bulbus cavernosum)

- Urethra
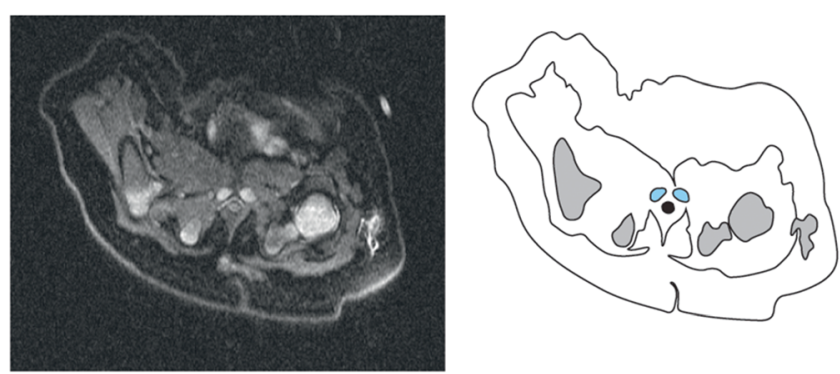

h

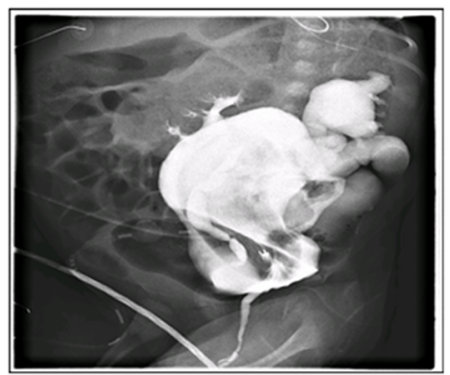

distal pouch or if perforation occurred at the proximal colos-

second event occurred during opacification of the distal bowel, but it was unclear if it was caused by perforation of the tomy during insertion of the Foley catheter. 
Eleven patients showed anomalies of the sacral vertebra visible on MRI, consisting of hypoplasia of the sacrum and/ or coccyx.

\section{Discussion}

In this study, we show that high resolution MR imaging of the pelvic floor is feasible in the majority of neonatal cases without the use of anaesthesia. This technique provides high-resolution images that allow the radiologist to delineate the various structures in ARM. To the best of our knowledge, the present study is the first comparison of high-resolution MRI findings, without MR fistulography, directly with colostography/fistulography on

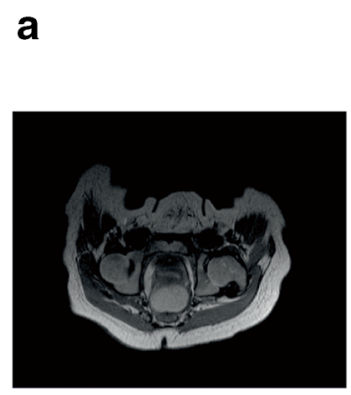

$$
\begin{aligned}
& \text { Bones } \\
& \text { Bowel } \\
& \text { Urethra } \\
& \text { Vagina }
\end{aligned}
$$

b
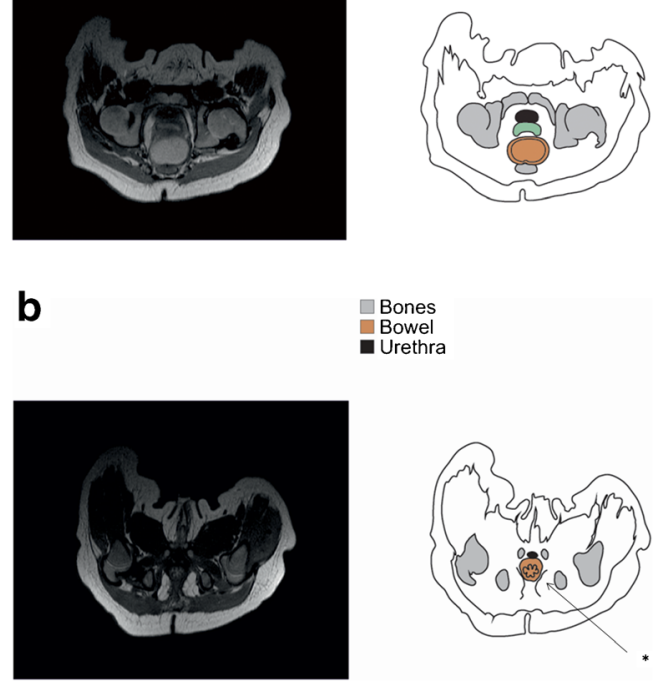

C

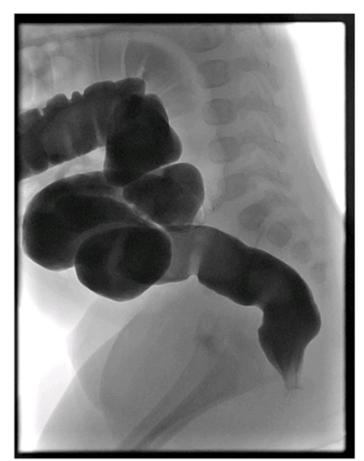

Fig. 2 One-month-old female neonate with a vestibular fistula (a-d). MRI and correlated drawing shows axial slices $(\mathbf{a}, \mathbf{b})$ of the distal orifice with typical layered aspect of the intrasphincteric region (like a starfish), which was therefore interpreted as a normal colon (b). This starfish sign $(*)$ refers to the layered aspect of the bowel on perpendicular view. $\mathbf{c}$ shows the anterior position of the rectal orifice on fistulography, with a normal composition of the intrasphincteric part, as in normal colon a side-by-side basis, taking surgery as a reference standard. We show that MRI is at least as good, but is also a better overall diagnostic tool than colostography/fistulography for classifying malformations and for the precise documentation of rectal pouch level in relation to the pelvic floor.

As colostography/fistulography is currently the option of choice in the preoperative setting, the primary aim of this study was to explore the non-minority state of an optimized MRI protocol in comparison to conventional imaging. Gross et al. stated that a posterior sagittal approach should never be attempted without a technically adequate high-pressure distal colostogram to determine the exact positions of the rectum and the fistula [14]. Based on our findings and findings from the literature, we conclude that MRI also fulfils these criteria, but with fewer risks of complications and a lower exposure to radiation.

Since an MRI examination is static (non-operator dependent) and images can always be re-evaluated and discussed with different physicians, MRI can produce more consistent results. Moreover, reconstructions and one to one comparison of images from various angles can be done at any moment. Very high-resolution sequences also allow multiplane reconstructions that provide additional insight into complicated anatomic anomalies.

Inter-reader agreement of conventional imaging was moderate to substantial, whereas MRI yielded perfect agreement. The main drawback in the interpretation of conventional imaging was the difficulty in differentiating prostatic from bulbar recto-urethral fistulas. This can be difficult since the location of the pelvic floor was not always clearly interpretable. Neither MRI reader had any difficulty in discerning these two entities, since adjacent structures such as the prostate made differentiation straightforward (Fig. 1). Operative techniques have evolved in recent years, and most bulbar recto-urethral fistulas with a rectal pouch at the pelvic floor are now treated with PSARP, whereas higher fistulas such as prostatic recto-urethral fistulas are more likely to be operated using LAARP. This development has made differentiation between these two ARM entities increasingly relevant for clinical practice. In light of the data presented here, we conclude that local anatomy is more precisely presented for surgical planning when using MRI compared to colostography/fistulography.

Based on results from the present cohort, liquid instillation in orifices appears to be unnecessary, contributing to the very low invasiveness of the procedure. The visualization of the different layers of the bowel wall (presenting with a typically serrated aspect) seems to be sufficient to differentiate between bowel and fistula (Fig. 2). This serration is probably caused by local contraction of the muscular layers giving it a starfish 
appearance, a well-known MRI aspect of a contracted bowel and anal sphincter muscle (Fig. 2). Preliminary data from two pilot studies suggested that MRI fistulography is as appropriate as conventional fistulography in the preoperative evaluation of ARM $[6,19]$. However, as all patients needed to be anaesthetized, disadvantages included invasiveness (and possible perforations), costs and time.

A major benefit of the MRI approach used in this study is that no anaesthesia or deep sedation was needed in the vast majority of our patient group. All parents were asked to feedand-wrap the patient, and the only case in which the quality of the MRI was uninterpretable was due to leg movements while crying. In this particular case, the parents had fed the baby some hours before, so hunger may have caused the restlessness.

Both MRI and colostography/fistulography accurately measured the length of the common channel in cases with cloacal malformation. The reference standard was surgery and urethrovaginoscopy. Additional information from colostography/ fistulography was only used if the investigation was clearly diagnostic and well performed, showing some additional value only when surgery and urethroscopy produced contradictory findings. We would argue that this is admissible because this approach leads to the most accurate length measurement. As there were only five patients with a cloaca, further studies are needed to build on these promising findings.

The main drawback of our study was the limited number of participants. However, recruiting a consecutive group of 36 patients at our tertiary centre required 5 years. Despite limited numbers, to the best of our knowledge, this is the first prospective study to date in which noninvasive MRI and colostography/fistulography are compared side-by-side. Further multicentre cohort studies are planned in order to allow firmer conclusions.

The high resolution of the images also provided an opportunity to analyse pelvic floor muscles. Since objectivising the reference standard from surgery was difficult, this information was not included. However, we assume that the current interpretation was more accurate than that of a previously published study from 2009, which utilized threefold thicker slices, but also more challenging, due to the younger median age of our patient group [20].

A final important drawback of our study was the retrospective analysis of the colostography/fistulography. Although both readers were experienced radiologists, prospective interpretation during data collection would have been preferable, but was unfortunately not possible due to organizational issues.

In conclusion, our results indicate that high resolution MRI without sedation is feasible and of great value in the diagnostic preoperative workup of patients with an ARM. We are confident that, when feasible, MRI will replace colostography/fistulography as the procedure of choice for ARM.

Acknowledgments The scientific guarantor of this publication is Maarten Thomeer. The authors of this manuscript declare no relationships with any companies. The authors of this manuscript declare no relationships with any companies, whose products or services may be related to the subject matter of the article. The authors state that this work has not received any funding. One of the authors has significant statistical expertise. No complex statistical methods were necessary for this paper. Institutional Review Board approval was obtained. Written informed consent was obtained from all subjects (patients) in this study. No subjects or cohorts have been previously reported. Methodology: prospective, diagnostic or prognostic study, performed at one institution.

Finally, we would like to acknowledge the high quality of the drawings made by Hans Kneefel.

Open Access This article is distributed under the terms of the Creative Commons Attribution-NonCommercial 4.0 International License (http:// creativecommons.org/licenses/by-nc/4.0/), which permits any noncommercial use, distribution, and reproduction in any medium, provided you give appropriate credit to the original author(s) and the source, provide a link to the Creative Commons license, and indicate if changes were made.

\section{References}

1. Wijers CH, van Rooij IA, Marcelis CL, Brunner HG, de Blaauw I, Roeleveld N (2014) Genetic and nongenetic etiology of nonsyndromic anorectal malformations: a systematic review. Birth Defects Res C Embryo Today 102:382-400

2. Alamo L, Meyrat BJ, Meuwly JY, Meuli RA, Gudinchet F (2013) Anorectal malformations: finding the pathway out of the labyrinth. Radiographics 33:491-512

3. Holschneider A, Hutson J, Pena A et al (2005) Preliminary report on the international conference for the development of standards for the treatment of anorectal malformations. J Pediatr Surg 40:1521-1526

4. Pena A (1986) Posterior sagittal approach for the correction of anorectal malformations. Adv Surg 19:69-100

5. Boemers TM, Beek FJ, Bax NM (1999) Review. Guidelines for the urological screening and initial management of lower urinary tract dysfunction in children with anorectal malformations-the ARGUS protocol. BJU Int 83:662-671

6. Kavalcova L, Skaba R, Kyncl M, Rouskova B, Prochazka A (2013) The diagnostic value of MRI fistulogram and MRI distal colostogram in patients with anorectal malformations. J Pediatr Surg 48:1806-1809

7. McHugh K, Dudley NE, Tam P (1995) Pre-operative MRI of anorectal anomalies in the newborn period. Pediatr Radiol 25: S33-S36

8. McHugh K (1998) The role of radiology in children with anorectal anomalies; with particular emphasis on MRI. Eur J Radiol 26:194199

9. Eltomey MA, Donnelly LF, Emery KH, Levitt MA, Pena A (2008) Postoperative pelvic MRI of anorectal malformations. AJR Am J Roentgenol 191:1469-1476

10. Nievelstein RA, Vos A, Valk J, Vermeij-Keers C (2002) Magnetic resonance imaging in children with anorectal malformations: embryologic implications. J Pediatr Surg 37:1138-1145 
11. Cremin BJ, Cywes S, Louw JH (1972) A rational radiological approach to the surgical correction of anorectal anomalies. Surgery 71:801-806

12. Lernau OZ, Nissan S (1980) Low anterior resection with a long posterior anorectal myectomy and sphincterectomy for Hirschsprung's disease. J Pediatr Surg 15:613-614

13. Podberesky DJ, Towbin AJ, Eltomey MA, Levitt MA (2013) Magnetic resonance imaging of anorectal malformations. Magn Reson Imaging Clin N Am 21:791-812

14. Gross GW, Wolfson PJ, Pena A (1991) Augmented-pressure colostogram in imperforate anus with fistula. Pediatr Radiol 21: 560-562

15. Whiting P, Rutjes AW, Reitsma JB, Bossuyt PM, Kleijnen J (2003) The development of QUADAS: a tool for the quality assessment of studies of diagnostic accuracy included in systematic reviews. BMC Med Res Methodol 3:25
16. Holschneider AM, Jesch NK, Stragholz E, Pfrommer W (2002) Surgical methods for anorectal malformations from Rehbein to Pena-critical assessment of score systems and proposal for a new classification. Eur J Pediatr Surg 12:73-82

17. Georgeson K (2007) Laparoscopic-assisted anorectal pull-through. Semin Pediatr Surg 16:266-269

18. Landis JR, Koch GG (1977) The measurement of observer agreement for categorical data. Biometrics 33:159-174

19. Alves JC, Sidler D, Lotz JW, Pitcher RD (2013) Comparison of MR and fluoroscopic mucous fistulography in the pre-operative evaluation of infants with anorectal malformation: a pilot study. Pediatr Radiol 43:958-963

20. Tang ST, Cao GQ, Mao YZ et al (2009) Clinical value of pelvic 3dimensional magnetic resonance image reconstruction in anorectal malformations. J Pediatr Surg 44:2369-2374 